\title{
Detection of brain hemorrhage in white matter using analysis of radio channel characteristics
}

\author{
Mariella Särestöniemi ${ }^{1}$, Carlos Pomalaza-Raez ${ }^{2}$, Jaakko Hakala ${ }^{3}$, Sami Myllymäki ${ }^{4}$, \\ Joni Kilpijärvi ${ }^{4}$, Jari Iinatti ${ }^{1}$, Matti Hämäläinen ${ }^{1}$, Teemu Myllylä ${ }^{3,5}$ \\ ${ }^{1}$ Centre for Wireless Communications, University of Oulu, Finland \\ ${ }^{2}$ Department of Electrical and Computer Engineering, Purdue University, USA \\ ${ }^{3}$ Optoelectronics and Measurement Techniques Research Unit, Faculty of Information \\ Technology and Electrical Engineering, University of Oulu, Finland \\ ${ }^{4}$ Microelectronics Research Unit, Faculty of Information Technology and \\ Electrical Engineering, University of Oulu, Finland \\ ${ }^{5}$ Research Unit of Medical Imaging, Physics and Technology, Faculty of Medicine, \\ University of Oulu, Finland \\ corresponding author: mariella.sarestoniemi@oulu.fi
}

\begin{abstract}
This paper presents a simulation -based study on detection of stroke/brain hemorrhage even in the white matter using radio channel characteristics analysis. The idea is to utilize the fact that blood has different dielectric properties than brain's white and grey matters and, thus, additional blood areas inside the brain change radio channel characteristics between the transmitter and receiver antennas located on the opposite sides of the head. The antennas should be strongly directive and designed to work attached to the body surface so that hemorrhages even in the white matter could be detected. The study is conducted using the electromagnetic simulation software CST and two different simulation models: a spherical tissue layer model and an anatomical voxel model. The antennas used in this study are bio-matched mini-horn antennas designed for implant communications at 1-4 GHz frequency range. Different sizes of the blood areas are evaluated. This initial study shows how even small sizes of hemorrhage can change radio channel even as the hemorrhage is located in the middle of the brain, in the white matter. The path loss difference is $0.5-10 \mathrm{~dB}$ between the hemorrhage and reference cases depending on the size and location of the hemorrhage. A practical solution of this hemorrhage detection technique could be a portable helmet type of structure having several small sized antennas around the internal part of the helmet. Such a helmet would be easy to use e.g. in ambulance, which would enable early detection of hemorrhage in its early phase and, hence, improve prospects of the cure significantly.
\end{abstract}

Keywords: Bio-matched horn antenna, brain hemorrhage, dielectric properties, early diagnosis, propagation loss, radio channel analysis. 


\section{Introduction}

Brain hemorrhage/stroke is a fatal physical condition, which may cause severe disability or even death, and thus it is essential to be diagnosed in its early phase. Current method for detecting hemorrhage and stroke in the brain is practically limited to computing tomography (CT) and magnetic resonance imaging (MRI). They are expensive and complex techniques and can be used only in central hospitals. Consequently, there is a strong need for a wearable and easy to use technique for fast detection of hemorrhage and stroke already outside hospital. In the literature, there are microwave -based studies presenting hemorrhage detection [1]-[12]. Most of them are based on detecting changes in the antenna return loss, which has restrictions. For instance, hemorrhage located deep inside the brain white matter cannot be detected reliably with return loss analysis. Besides, several evaluations in the literature are conducted with the large antennas, which are not suitable for practical portable applications. The idea of helmet type brain hemorrhage detection device was originally presented in [13] which would measure channel parameters between the antennas located in different sides of the head. However, Ref. [13] presents only the idea, not any measurement or simulation results.

Most of the simulation -based studies in the literature are conducted using simplified head models. There is a lack of studies presenting radio channel characteristics -base brain hemorrhage detection, which would have been conducted using a realistic voxel model and directive antennas. Besides, there is lack of studies presenting brain hemorrhage detection in the white matter.

This study focuses detecting brain hemorrhage in the white matter in the middle part of the brain, since it is considered the most challenging part for hemorrhage detection. The paper involves propagation studies in the head area aiming at detection of hemorrhage and stroke using radio channel characteristics analysis. Hemorrhage detection could be done by using a simple, portable helmet type of monitoring device in which several transmitter and receiver antennas are located inside the helmet. The diagnosis could be done by setting the helmet in the patient's head, measuring and analysing the radio channel characteristics, and hence the diagnosis could be done outside the hospital in ambulance or even in patient's home which is important for rural area health care. Hence, the medical treatment could be started immediately, and prospects of cure could be significantly improved.

The physical phenomenon behind the proposed technique is that blood have different dielectric properties than the brain matter (grey and white), as shown in Table I [14]. Especially the relative permittivity of the blood is significantly higher than that of the brain matters. Thus, blood area inside brain change propagation and channel characteristics between the transmitter and receiver antennas, which are located in different sides of the head. Since the propagation losses in the tissues are relatively high, strongly directive antennas should be used so that the received signal is on the level in which the changes caused by hemorrhage can be still detected.

The objective of this paper is to present electromagnetic simulations -based study on the detection of brain hemorrhage in white matter using radio channel characteristics analysis. CST Simulia Studio Suite [15], which is based on finite integration technique, is used in the simulations. CST's anatomical voxel model Hugo is chosen for these 
evaluations since it has detailed brain structure with separate grey and white matters. The aim is to show how brain hemorrhage with different sizes and different locations can be detected using a directive on-body antenna designed for in-body communications. Even hemorrhages in the middle of the white matter can be detected with this method. 2D power flow illustration and power values at different locations inside the brain of the voxel show how the blood areas change the propagation. Besides, power loss calculations are presented to show the difference between the hemorrhage and reference cases.

The paper is organized as follows: Section II presents Study case by describing the antenna and simulation model used in this study. Power flow presentation are illustrated in Section III. Channel evaluations are presented in Section IV, and Conclusions are given in Section V.

Table I. Dielectric properties of different tissues in the head.

\begin{tabular}{llll}
\hline \multicolumn{4}{c}{ Dielectric properties at selected frequencies } \\
\cline { 2 - 4 } & $1 \mathrm{GHz}$ & $2 \mathrm{GHz}$ & $4 \mathrm{GHz}$ \\
& Relative Permittivity & Relative Permittivity & Relative Permittivity \\
Tissue & $/$ Conductivity $[\mathrm{S} / \mathrm{m}]$ & $/$ Conductivity $[\mathrm{S} / \mathrm{m}]$ & $/$ Conductivity $[\mathrm{S} / \mathrm{m}]$ \\
\hline Skin & $40.9 / 0.9$ & $38.6 / 1.27$ & $36.6 / 2.34$ \\
Fat & $11.3 / 0.12$ & $11.0 / 0.12$ & $10.4 / 0.50$ \\
Muscle & $54.8 / 0.98$ & $53.3 / 1.45$ & $50.8 / 3.02$ \\
Skull bone & $20.6 / 0.36$ & $19.1 / 0.65$ & $16.9 / 1.40$ \\
Cerebrospinal fluid & $68.4 / 2.46$ & $66.9 / 3.07$ & $63.7 / 5.20$ \\
Brain grey matter & $52.3 / 0.99$ & $49.7 / 1.51$ & $46.6 / 3.09$ \\
Brain white matter & $38.6 / 0.62$ & $36.7 / 1.00$ & $34.5 / 2.14$ \\
Blood & $61.1 / 1.58$ & $59.0 / 2.19$ & $55.7 / 4.13$ \\
\hline
\end{tabular}

\section{Study case}

\subsection{Directive on-body antenna}

In this study, a directive bio-matched mini-horn antenna illustrated in Fig.1, is used in the evaluations. The antenna is originally presented in [16] and based on the documentation it was remodeled for this study case. The dimensions of the antenna were further modified slightly to get better matching with the voxel model. The modified structure has dimension $\mathrm{h}=2.7 \mathrm{~cm}$ and $\mathrm{d}=1.8 \mathrm{~cm}$. The bio-matched horn antenna is composed of water-filled holes, which mimic the frequency-dependent relative permittivity of the underlying tissue over its entire bandwidth. Details of the antenna structure can be read in [16].

The modified antenna structure is tuned to operate at $1.7-3.3 \mathrm{GHz}$. The antenna's $\mathrm{S}_{11}$ parameter is presented in Fig. 2. 


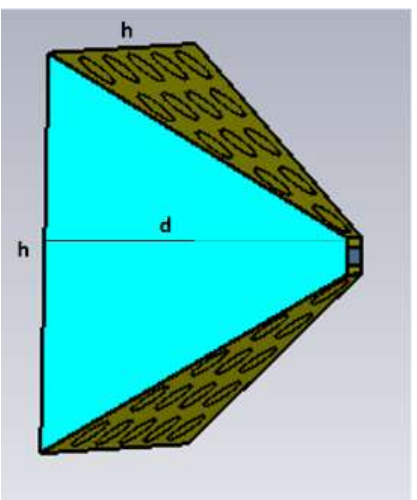

Fig. 1. A mini horn antenna designed for in-body communications.

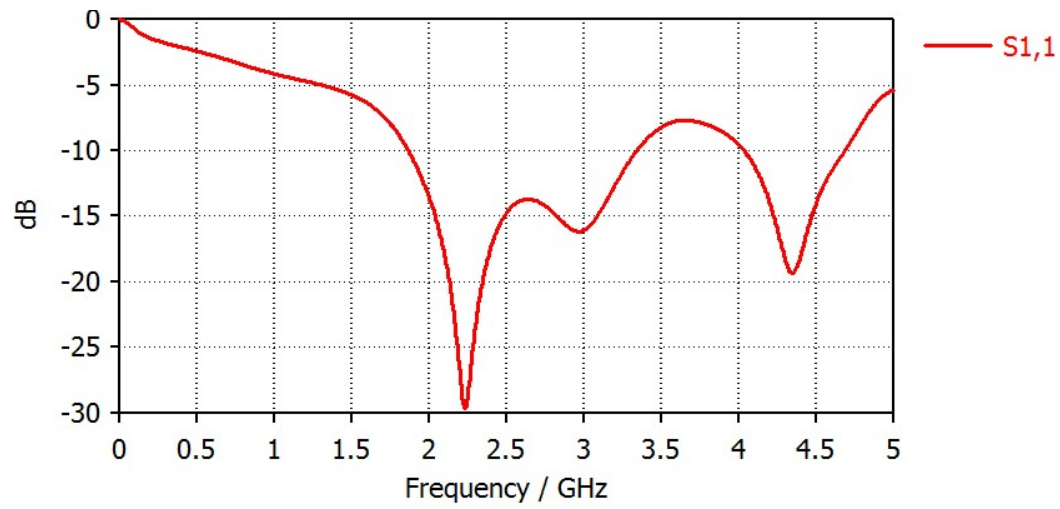

Fig. 2 Reflection coefficient $\mathrm{S}_{11}$ of the mini horn antenna as located on the skin.

\subsection{Simulation models}

The simulations were carried out using the CST studio suite, which is based on the finite integration technique. Two different simulation models were used in this study: a spherical tissue layer model and CST's anatomical Hugo voxel model, which are presented in Figs. 3a-b, respectively. The spherical layer model is a full sphere, which consists of several different nested tissue spheres, each layer having realistic thicknesses. The thicknesses are presented in Table II. The anatomical voxel model Hugo has the detailed brain model with separate grey and white matters having realistic shapes. The resolution of the Hugo voxel is $1 \mathrm{~mm} \times 1 \mathrm{~mm} \times 1 \mathrm{~mm}$.

Antennas are located on the skin in the middle of the head, as shown in Fig. 4. For this study case, we chose the widest part in the head where the muscle layer is thickest since it can be seen as to the most challenging location in terms of radio signal propagation. The diameter of the voxel model's head is $16 \mathrm{~cm}$ in this location and thus also the diameter of the spherical model is set $16 \mathrm{~cm}$. 
Bio-matched mini-horn antennas are designed to work so that they are completely attached to the skin [16]. Although the resolution of Hugo voxel is small, pixelization may have strong impact on the antenna and channel characteristics, as explained in [17]. Thus, the non-smoothness of the voxel skin surface is compensated by inserting thin skin layer in front of the antenna so that it overlaps with the voxel's non-smooth skin surface.

The possibility to detect brain hemorrhage is studied by inserting blood pieces of different sizes in the voxel model's brain white matter. The sizes with the corresponding dimensions are summarized in Table II. The blood pieces and their locations are presented in Fig. 4 for the spherical layer model and in Fig. 5 for the voxel model. The spherical model is evaluated with the large piece in two different locations, whereas the voxel model is evaluated with large, medium, and small pieces, which are located in the middle of the brain.

a)

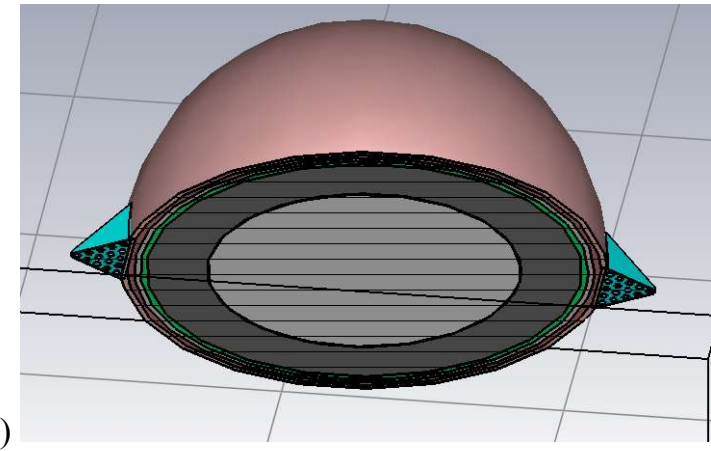

b)

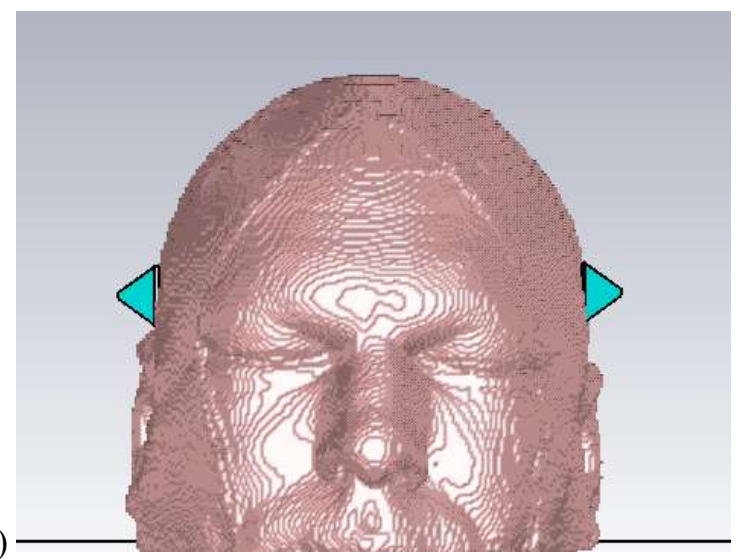

Fig. 3. a) Spherical layer model and b) Hugo voxel model with horn-antennas placed on the skin. 
a)

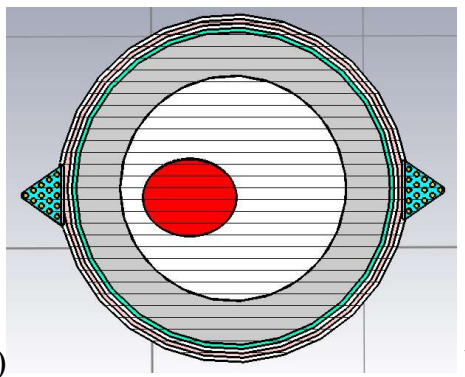

b)

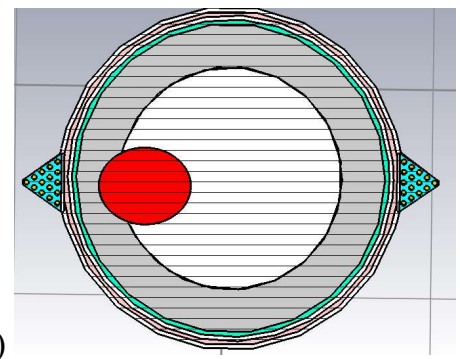

Fig. 4. Blood areas on the spherical model a) middle, b) left.

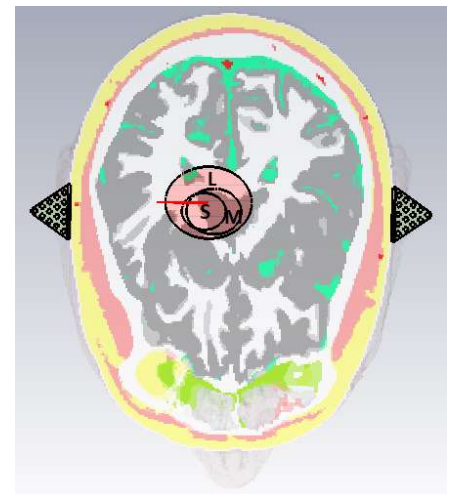

Fig. 5. Different sizes of brain hemorrhages in the cross-cut of the voxel model.

Table II. Dimensions of different sizes of brain hemorrhages.

\begin{tabular}{llll}
\hline Tissues & Small $(\mathrm{S})$ & Medium $(\mathrm{M})$ & Large $(\mathrm{L})$ \\
\hline Dimensions $(\mathrm{x}, \mathrm{y}, \mathrm{z})$ & $(2,2,1) \mathrm{cm}$ & $(3,2,1) \mathrm{cm}$ & $(5,4,2) \mathrm{cm}$ \\
\hline
\end{tabular}

\section{Power flow analysis}

In this section, the power flow from the transmitter antenna to the receiver antenna is presented to understand how the hemorrhage change the propagation inside the brain tissues. Power flow representation at $1.7 \mathrm{GHz}$ with and without the hemorrhage are presented in Figs. 6a-b, respectively. The $\mathrm{dB}$ range for the power flow presentation is $0--65 \mathrm{~dB}$, where the reference $0 \mathrm{~dB}$ level is set to the antenna. Power flow on the skin is $-26 \mathrm{~dB}$.

From Figs. 6a-b one can note clear differences between the cases with and without the hemorrhage. In the presence of hemorrhage, the power flow is narrower. Within the plotted $\mathrm{dB}$ range, the $\mathrm{Rx}$ antennas is achieved easily in the reference case, whereas in the case of hemorrhage, the Rx antenna is hardly achieved. The differences in the power flows are due to the differences between the dielectric properties between the blood and 
brain tissues. Blood has clearly higher permittivity than the brain matters (grey/white) and thus, the power loss is larger in the presence of hemorrhage.

Table III presents the comparison between the power loss values obtained with the reference model and the hemorrhage model in the locations depicted in Figs. 6c. In the location A, which is close to the Rx antenna, the instant power flow value for the reference model at the selected cross-cut is $2 \mathrm{~dB}$ higher than that of the hemorrhage model. In point $\mathrm{B}$, the power loss with the hemorrhage model is $6.5 \mathrm{~dB}$ higher than with the reference model. Interestingly, at point $\mathrm{C}$, the reference model has higher power loss although the power inside the blood area is expected to be smaller. However, at point $\mathrm{D}$, there is no difference between the hemorrhage and reference cases. Instead, at the point $\mathrm{E}$, the difference is the power loss being higher with the hemorrhage model. It is interesting to note that the blood properties affect the power inside the hemorrhage, and this requires further studies.
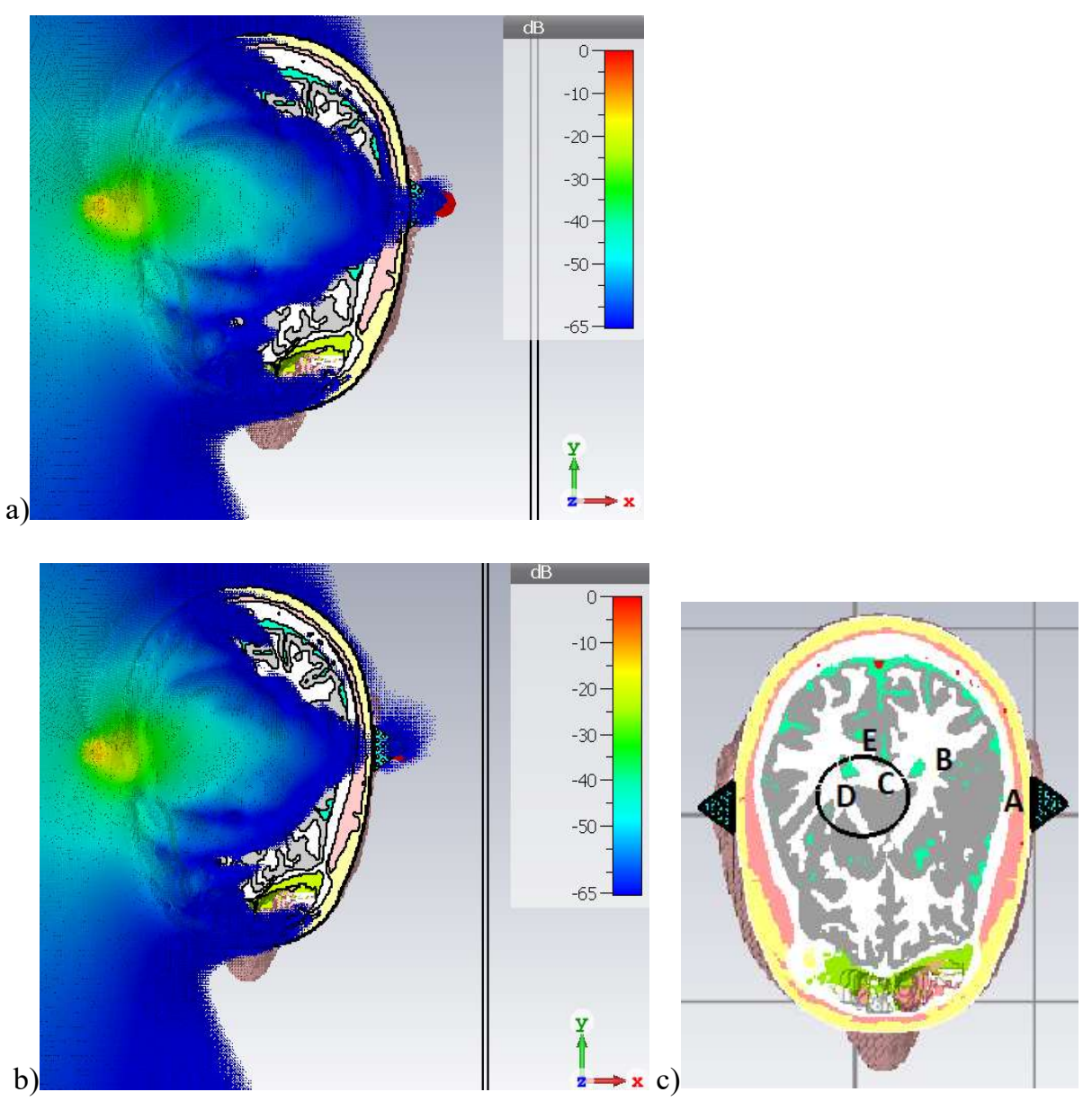

Fig. 6. Power flows at $1.7 \mathrm{GHz}$ a) in the reference case, b) in the hemorrhage case, c) points A$\mathrm{E}$ where power is measured. 
Table III. Power measured in points A-E at $1.7 \mathrm{GHz}$.

\begin{tabular}{llllll}
\hline $\begin{array}{l}\text { Power at } \\
\text { points }\end{array}$ & $\mathrm{A}[\mathrm{dB}]$ & $\mathrm{B}[\mathrm{dB}]$ & $\mathrm{C}[\mathrm{dB}]$ & $\mathrm{D}[\mathrm{dB}]$ & $\mathrm{E}[\mathrm{dB}]$ \\
\hline Reference & -71.6 & -71.5 & -54 & -48 & -53 \\
Hemorrhage & -73.6 & -77.1 & -52 & -48 & -56 \\
\hline
\end{tabular}

As a comparison, power loss is calculated with Matlab based on planar model propagation. The calculation utilises an extension of the algorithm that includes lossy media proposed by Orfanides [16]. The tissue thicknesses as well as calculated power loss values in the presence of large size hemorrhage are presented in Table IV. As one can note, the power loss difference is significant between the hemorrhage and the reference case: in the presence of the hemorrhage having a large size, the loss is $9 \mathrm{~dB}$ higher at 1.7 GHz. However, in the power flow evaluations presented above, the difference is milder. The difference is due to the more complex structure of the voxel model, which enables alternative propagation paths than just a direct path through the tissues, which are on the direct line between the transmitter and the receiver antennas. However, power loss values obtained using Matlab provides good reference showing clearly the impact of the blood on the channel characteristics.

Table IV. Thicknesses of the layers used in the power loss calculations.

\begin{tabular}{llll}
\hline Tissues & relative permittivity & loss tangent & thickness [mm] \\
\hline Air & 1 & 0 & 0 \\
Skin Dry & 39,04 & 1,146 & 0,002 \\
Muscle & 53,68 & 1,288 & 0,006 \\
Skull & 15,665 & 0,408 & 0,002 \\
CSF & 67,34 & 2,853 & 0,002 \\
Grey-Matter & 50,29 & 1,334 & 0,015 \\
White-Matter & 37,48 & 0,8737 & 0,04 \\
Blood & 59,55 & 1,976 & 0,05 \\
White-Matter & 37,48 & 0,8737 & 0,016 \\
Grey-Matter & 50,29 & 1,334 & 0,015 \\
CSF & 67,34 & 2,853 & 0,002 \\
Skull & 15,665 & 0,408 & 0,002 \\
Muscle & 53,68 & 1,288 & 0,006 \\
Skin Dry & 39,04 & 1,146 & 0,002 \\
Air & 1 & 0 & 0 \\
Power loss in the reference case: $50 \mathrm{~dB}$ & & \\
Power loss with blood: $59 \mathrm{~dB}$ & & \\
\hline
\end{tabular}




\section{Channel characteristics analysis}

First, the channel characteristics are evaluated with the spherical model in the presence and absence of blood. Channel parameters $S_{21}$ s obtained using the reference model and the hemorrhage model are presented in Fig. 7. Hemorrhage location middle and left are included. As it can be seen, blood in both brain areas cause clear differences in the channel characteristics, especially in the frequency range $2.5-4 \mathrm{GHz}$. However, at lower frequencies $1.3-2 \mathrm{GHz}$ one can note differences in the channel responses as well though the differences are milder, approximately $1.5 \mathrm{~dB}$ in this case. The closer the modelled hemorrhage area is to the antenna, the clearer is the difference. Interestingly, $\mathrm{S}_{21}$ obtained with the reference model is at clearly higher level than $\mathrm{S}_{21}$ of the models with blood pieces only in the frequencies $2.5 \mathrm{GHz}$ onwards. At lower frequencies it is vice versa at certain frequencies. When comparing the dielectric properties of the blood, grey and white matter, one can note that within the simulated frequency range, the relativity permittivity of the blood is clearly higher than those of brain matters. Instead, the conductivity values are higher for the blood area.

Next, the $S_{21}$ s obtained using the voxel model in the reference case and hemorrhage case are compared. The results are presented in Fig. 9. As it can be seen, the $S_{21}$ of the reference model is at higher level than the $\mathrm{S}_{21}$ of the hemorrhage model at most of the frequencies, as expected from the power loss calculations. However, at e.g. $2.6 \mathrm{GHz}$, the $S_{21}$ of the reference case is at lower level and the case of largest hemorrhage is at highest level. Obviously, the difference on dielectric properties of blood and brain matters have variation depending on the frequency. Thus, it is important to select carefully the frequency range used for this technique.

The zoomed version of the $S_{21}$ results at lower frequencies are presented in Fig. 9. As one can note, even at lower frequencies the difference is $1-5 \mathrm{~dB}$, which is clearly detectable in the practical solutions.

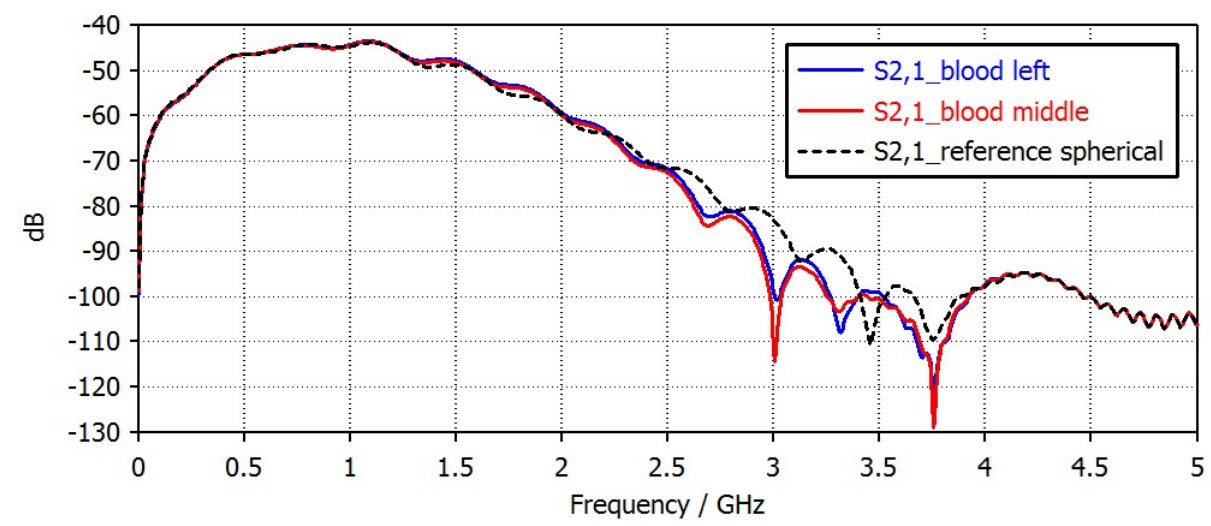

Fig. 7. $\mathrm{S}_{21}$ comparison with the spherical layer model as the hemorrhage is in the middle of the brain and in the left part of the brain. 


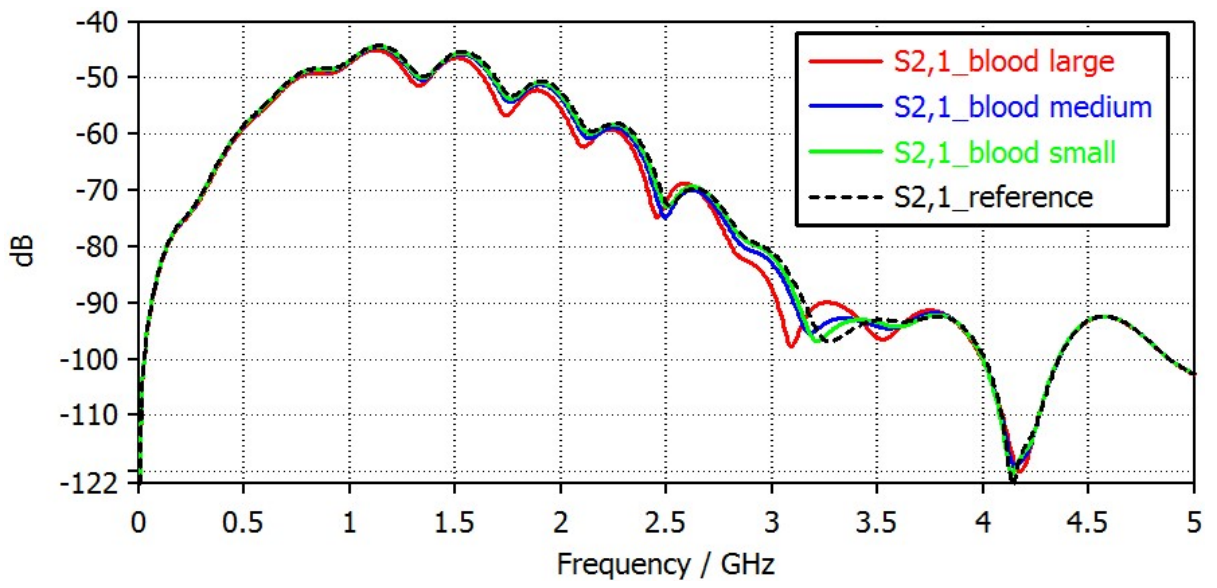

Fig. 8. $\mathrm{S}_{21}$ channel parameters obtained using the voxel model with different sizes of hemorrhage.

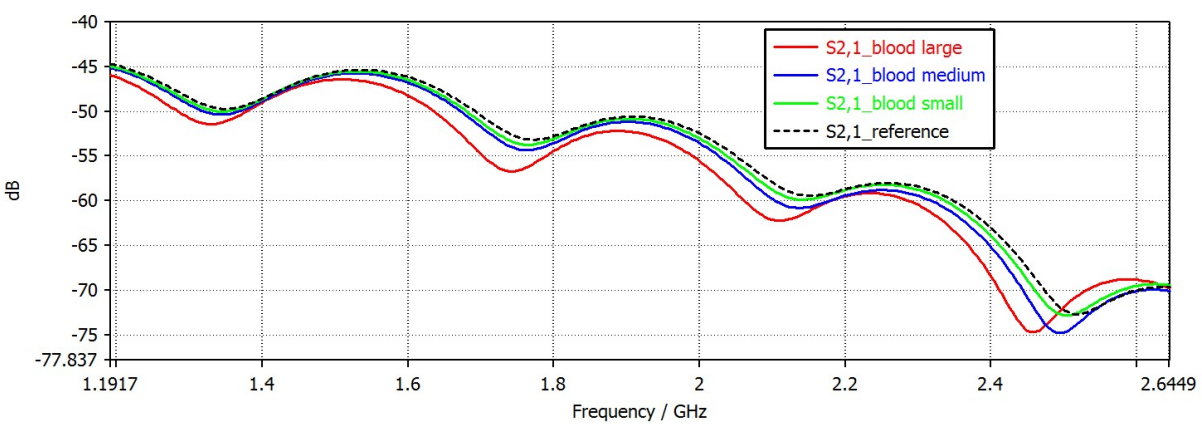

Fig. 9. Zoomed version of $\mathrm{S}_{21}$ at lower frequencies.

\section{Conclusions}

Detection of the brain hemorrhage in its early face is essential for the better prospects of cure. This paper presented a simulation -based study of detecting brain hemorrhage in the white matter using radio channel characteristics analysis, which could be realized in a portable and compact helmet type of structure with several small mini-horn antennas inside. The study is conducted by using the electromagnetic simulation software CST and two different simulation models: a spherical tissue layer model and an anatomical voxel model. The bio-matched mini-horn antennas designed for implant communications were used in this study.

The idea of this technique was to utilize the fact that blood has different dielectric properties than brain's white and grey matters and, thus, additional blood areas inside the brain change the radio channel characteristics between the transmitter and receiver antennas located on opposite sides of the head. This initial study showed how even 
small sizes of hemorrhage can change the radio channel characteristics even when hemorrhage is located in the middle of the brain, in the white matter. The path loss difference was $0.5-15 \mathrm{~dB}$ between the reference and hemorrhage cases depending on the frequency.

Power flow studies at $1.7 \mathrm{GHz}$ revealed interesting phenomenon: although the power loss is expected to be larger in the presence of blood, the power inside the certain part of the hemorrhage area was noted to be higher than in the respective location without the blood. This is one of the future topics we are aiming to solve. Besides, other future work objectives include more detail studies on the impact of the hemorrhage in different frequencies and with several antennas to maximize visibility of the difference. Optimal antenna locations will be determined as well. Furthermore, different sizes of the head models will be used in the studies. Moreover, radio channel measurements will be conducted using a real human skull filled with phantom liquids [17] for brain matter and blood and prototypes of the bio-matched mini-horn antenna.

A practical solution of this hemorrhage detection technique could be a helmet type of structure having several small sized antennas around the internal part of the helmet. The brain hemorrhage could be detected analyzing channel characteristics of different antenna combinations. The benefit of helmet type of brain hemorrhage detector would be its easy use e.g. in ambulance. This would enable the early detection of hemorrhage in its early phase and hence, improve prospects of the cure significantly even for the patients living in rural areas far away from the hospitals.

The side effects and risks related to the radio channel analysis -based hemorrhage detection techniques are minor since the measurement itself is very fast and thus, the exposure for radiation is minor. Besides, input power can be kept low to meet the Specific Absorption Requirements (SAR) for the antennas.

\section{Acknowledgement}

This research has been financially supported by the project WBAN Communications in the Congested Environments and in part by Academy of Finland 6Genesis Flagship (grant 318927) and Academy of Finland grant 318347. This project has received funding from the European Union's Horizon 2020 research and innovation program under the Marie Sklodowska-Curie grant agreement No. 872752

Bio-matched mini-horn antenna was re-modeled by Mikko Parkkila and Uzman Ali from Radientum, which is greatly acknowledged.

\section{References}

1. Dang-Dang Wang, Dan-Yang Chen, Hua-Shan Luan, Hong-Li Peng, "A New Method for Transcranial Vortex Microwave Beam Imaging", Microwave and Millimeter Wave Technology (ICMMT) 2018 International Conference on, pp. 1-3, 2018. 
2. A. Abbosh, "Microwave systems for head imaging: Challenges and recent developments," 2013 IEEE MTT-S International Microwave Workshop Series on RF and Wireless Technologies for Biomedical and Healthcare Applications (IMWS-BIO), Singapore, 2013, pp. 1-3, doi: 10.1109/IMWS-BIO.2013.6756184.

3. A. Zamani, A. M. Abbosh and A. T. Mobashsher, "Fast Frequency-Based Multistatic Microwave Imaging Algorithm With Application to Brain Injury Detection," in IEEE Transactions on Microwave Theory and Techniques, vol. 64, no. 2, pp. 653-662, Feb. 2016, doi: 10.1109/TMTT.2015.2513398.

4. A. T. Mobashsher, A. Mahmoud and A. M. Abbosh, "Portable wideband microwave imaging system for intracranial hemorrhage detection using improved back-projection algorithm with model of effective head permittivity," Scientific Reports, vol. 6, no. 20459,pp. 1-17, 2016.

5. J. Li, H. L. Peng, B. Q. Zong and Z. Yu, "A dual-polarized impulse radiation antenna for cerebral hemorrhage detection applications," 2014 IEEE MTT-S International Microwave Workshop Series on RF and Wireless Technologies for Biomedical and Healthcare Applications (IMWS-Bio2014), London, 2014, pp. 1-3.

6. D. Huang, H. L. Peng, X. Li, W. Jin and J. Mao, "A near-field spherical layered human head model for stroke detections,"Asia-Pacific Microwave Conference (APMC), Nanjing, 2015, pp. 1-3.

7. E. C. Fear, X. Li, S. C. Hagness and M. A. Stuchly, "Confocal microwave imaging for breast cancer detection: localization of tumors in three dimensions," in IEEE Transactions on Biomedical Engineering, vol. 49, no. 8, pp. 812-822, Aug. 2002.

8. D. Ireland, M. Bialkowski, "Microwave head imaging for stroke detection," Progress in Electromagnetics Research, Vol. 21, s.163-175, 2011.

9. Z. Tang, H. L. Peng, Y. Zhong and J. F. Mao, "A novel antenna array for stroke enhanced detection," 2015 Asia-Pacific Microwave Conference (APMC), Nanjing, 2015, pp. 1-3.

10. H. Yousefi Mesri, "Localization of hemorrhage site in stroke patients using multichannel microwave measurements," 2012 Annual International Conference of the IEEE Engineering in Medicine and Biology Society, San Diego, CA, 2012, pp. 5927-5930, doi: 10.1109/EMBC.2012.6347343.

11. A. E. Stancombe, K. S. Bialkowski and A. M. Abbosh, "Portable Microwave Head Imaging System Using Software-Defined Radio and Switching Network," in IEEE Journal of Electromagnetics, RF and Microwaves in Medicine and Biology, vol. 3, no. 4, pp. 284-291, Dec. 2019, doi: 10.1109/JERM.2019.2901360.

12. A. T. Mobashsher, A. M. Abbosh and Y. Wang, "Microwave System to Detect Traumatic Brain Injuries Using Compact Unidirectional Antenna and Wideband Transceiver With Verification on Realistic Head Phantom," in IEEE Transactions on Microwave Theory and Techniques, vol. 62, no. 9, pp. 1826-1836, Sept. 2014, doi: 10.1109/TMTT.2014.2342669.

13. A. Fhager and M. Persson, "Stroke detection and diagnosis with a microwave helmet," 2012 6th European Conference on Antennas and Propagation (EUCAP), Prague, 2012, pp. 17961798, doi: 10.1109/EuCAP.2012.6206715.

14. https://www.itis.ethz.ch/virtual-population/tissue-properties/database/dielectric-properties.

15. J. Blauert, A. Kiourti, "Bio-matched horn: A novel 1-9 GHz on-body antenna for low-los biomedical telemetry with implants," IEEE Transaction on antennas and propagation, vol. 67, no. 8, August 2019.

16. J. Orfanidis, "Electromagnetic Waves and Antennas," 2002, revised 2016, online: http://www.ece.rutgers.edu/ orfanidi/ewa/. 
17. J. Hakala, J. Kilpijärvi, M. Särestöniemi, M. Hämäläinen, S. Myllymäki and T. Myllylä, "Microwave Sensing of Brain Water - a Simulation and Experimental Study Using Human Brain Models," IEEE Access, vol. 8, pp. 111303-111315. 